\title{
Comparison of Knee Muscle Strength and Endurance of Affected Legs of Korean Women Diagnosed with Kellgren-Lawrence grade (K-L II) by Age
}

\author{
Soung-Kyun Hong $\cdot$ Dong-Geon Lee $\cdot$ Sun-Hae Song $\cdot$ Seung-Hoo Lee $\cdot$ Doo-Chul Shin ${ }^{1 \dagger}$
}

Department of Physical Therapy, Graduate School of Kyungnam University, Changwon, Republic of Korea ${ }^{1}$ Department of Physical Therapy, Kyungnam University, Changwon, Republic of Korea

Received: July 8, 2019 / Revised: July 16, 2019 / Accepted: August 31, 2019

(c) 2019 J Korean Soc Phys Med

\section{| Abstract |}

PURPOSE: The purpose of this study was to compare the maximum knee strength and endurance of elderly Korean women with Kellgren-Lawrence grade (K-L II) by age.

METHODS: In this study, the total number of participants was 50 , divided into five groups of 10 by age. Maximum knee muscle strength was measured at a low speed of $60 \% \mathrm{sec}$ using Biodex, an isokinetic device for comparison of maximum knee muscle strength, whereas muscle endurance was measured by muscle contraction at a high speed of $180^{\circ} / \mathrm{sec}$. The peak torque values of the extensor and flexor muscles in the muscle strength and muscle endurance tests were collected according to each classification.

RESULTS: The results of the experiment show that maximum muscle strength and muscle endurance decreased significantly at $60 \% \mathrm{sec}$ and $180 \% \mathrm{sec}$ during flexion and extension according to age $(p<.05)$. In particular, the age

†Corresponding Author : Doo-Chul Shin

icandox77@kyungnam.ac.kr, https://orcid.org/0000-0002-8873-9541

This is an Open Access article distributed under the terms of the Creative Commons Attribution Non-Commercial License (http://creativecommons.org/licenses/by-nc/3.0) which permits unrestricted non-commercial use, distribution, and reproduction in any medium, provided the original work is properly cited. group of 71 to 75 years showed the most significant correlation with other age groups $(p<.05)$.

CONCLUSION: The results of this study can be used as basic data for increasing physical activity and muscle strength in women with knee osteoarthritis.

Key Words: Muscle strength, Osteoarthritis, Knee, Aging

\section{Introduction}

Due to increasing medical and economic development around the world, research interest in aging has accelerated. Musculoskeletal problems associated with aging include tendon and ligament calcification and muscle fiber changes [1], which weaken muscle strength and muscle endurance, leading to limitations in daily life [2]. Arthritis is most commonly seen in weight-bearing joints, and it commonly occurs in the knees [3]. Patients with knee arthritis are limited in their daily lives by chronic pain [4]. During bouts of knee pain, the joint space is reduced in the bones and joints. In the soft tissues, increased viscoelasticity and shrinkage factors lead to shortening and weakening of the muscle quadratus femoris and back thigh muscle, resulting in a reduced motion range [5]. In addition, patients with knee osteoarthritis have been reported to have reduced total 
momentum, muscle torque, muscle strength, and resistance in the muscle quadratus femori [6]. Another study that examined 16 patients who underwent unilateral knee arthroplasty and measured activity quantities in the unaffected leg and unaffected knee extensor and flexor muscles reported that the strength of the muscle quadratus femoris was $60 \%$ compared to the unaffected knee, and the peak torque value and total activity during afferent and efferent contraction were reduced by $1 / 3$ compared to the affected leg [7].

Light to moderate K\&L grade 1-2, classified by Kellgren and Lawrence [8], exhibits a $10 \%$ loss of cartilage or cartilage tear at mild levels [8], limited knee joint flexion and extension, and reduction of muscle endurance to adjust the joint position [9]. Possible muscle weakening at this stage leads to difficulty in directional control of the joint [10] as well as loss of the proprioceptive sense of the knee joint [11], which results in reduction of postural control ability, an essential ability to prevent falling injuries $[12,13]$.

It is also known that degenerative knee osteoarthritis is associated with a higher incidence rate in females than males [14], and according to another study, the data showed a strong correlation between osteoarthritis and age [15].

Factors affecting the function of the knee have been reported to be sex [16,17], age [18], obesity [19], daily lifestyle [18], muscular exercise [20] etc., and these various factors are associated with kinetic differences such as a larger angle in women for Q-angle and genu recurvatum of the knees [21]. In addition, specific habits and behaviors in daily life that vary by culture are related to risk of disease or damage to the body. Oriental culture involves a lot of knee-bending and deep bowing in daily life, and this can cause overloading of the knees. Especially for aged Asian people who perform this movement repeatedly as they get older, the occurrence rate of arthritis in the knees is very high [22]. In addition, women have lower knee strength enhancement rates compared to men, and women have a lower EMG amplitude than men when walking down the stairs, thereby reducing muscle strength activation and delaying response time [17]. These kinetic characteristics result in a higher rate of knee soft tissue damage in women than in men.

In recent studies, there have been several studies on the correlation between incidence of degenerative knee arthritis and general characteristics such as age and sex [16-18], but there have been almost no comparative studies on Korean women with knee arthritis, physical characteristics according to age, muscular strength, and endurance of affected legs. Therefore, this study investigated the characteristics of knee osteoarthritis, physical characteristics according to age, and muscle strength and endurance of affected legs among Korean women diagnosed with light to moderate knee arthritis (KL grade II).

\section{Methods}

\section{Participants}

This study was conducted as a cross-sectional survey study. In this study, a total of 50 patients with knee arthritis diagnosed with Kellgren and Lawrence grade 2 (K-L II) were selected, and 10 participants were divided into five groups by the age of 5 years [23]. The selection criteria of the subjects were as follows: 1) a woman over 55-years-old, 2) a person diagnosed with osteoarthritis in only one side, 3) a person who has not received artificial joint replacement in the knee joint, 4) a person who can walk more than $10 \mathrm{~m}$ independently, 5) a person who has no inherent structural malformation or deformity that may affect walking and strength. The exclusion criteria were as follows: 1) a person who may suffer cardiovascular, respiratory, neurological, or musculoskeletal complications during maximal vasoconstriction testing and 2) a person with a grade of 5 or higher on the visual analog scale (VAS). Data on the subjects were collected from January 
2016 to December 2016. If the target number (10 persons) per age group was satisfied, no further recruitment was made. This study was approved by the Institute Review Board of the Bumin Hospital, Haeundae (201804CTDG-009).

\section{Procedure}

This study retrospectively examined the medical records of the subjects. Age, height, and body weight were measured. Body mass index was calculated by body weight (kg) / height $\left(\mathrm{m}^{2}\right)$.

The Biodex system (Biodex System 3 Pro, Biodex Medical Systems, Inc, New York, USA) was used to collect information such as motion range of knee joints and the maximum strength of voluntary muscles. This study was based on the standard procedure of the Biodex system [24]. The subjects' thighs, shins, and pelvises were fixed by a Biodex sheet and then bent at a $90^{\circ}$ angle. The complete extension of the knee was regarded as $0^{\circ}$, and $90^{\circ}$ knee flexion was regarded as a parameter for knee flexion. The affected knee was forcibly flexed to $90^{\circ}$, and the affected knee was completely lowered while the isokinetic movement was attempted. The isokinetic strength of the knee joint was measured by measuring the flexor and extensor muscles of the knee joint at low speed $\left(60^{\circ}\right)$ and high speed $\left(180^{\circ}\right)$. The maximum muscle strength was measured by muscle contraction at a low speed of $60 \% \mathrm{sec}$, and muscle endurance was measured by muscle contraction at a high speed of $180^{\circ} / \mathrm{sec}$. Three sets of measurements were made by starting from dorsiflexion through flexion to extension as one set five times at a measurement load speed of $60 \% \mathrm{sec}$, and three sets of measurements were made by starting from dorsiflexion through flexion to extension as one set five times at a measurement high speed of $180^{\circ} / \mathrm{sec}$. The break time between sets was set to 60 seconds [25]. The unit of motion range is degrees, and the peak torque value was measured and analyzed using $\mathrm{N}-\mathrm{M}$ (Newton-Meter). The average value of the measured values was used. The test-retest reliability ICC of the instrument used in the evaluation was very high at .75-.98.

\section{Statistical Analysis}

Statistical analysis of this study was performed using SPSS 18.0 software. The general characteristics of the subjects and the regularity test of muscle strength and endurance of the legs with degenerative arthritis were analyzed using Kolmogorov-Smirnov. As a result, normal distribution was not available, so nonparametric statistics were used. The Kruskal-Wallis test was used for muscle strength and endurance of each age group, and the Mann-Whitney test was used for the post-test. The significance level was set at .05 .

\section{Results}

\section{General Characteristics of the Subjects}

Participants were women aged over 55 years, and a total of 50 persons were subjects. The general characteristics of the subjects are shown in Table 1 .

Table 1. General Characteristic of Subjects

\begin{tabular}{cccccc}
\hline \multirow{2}{*}{ Age (year) } & $56-60(\mathrm{n}=10)$ & $61-65(\mathrm{n}=10)$ & $66-70(\mathrm{n}=10)$ & $71-75(\mathrm{n}=10)$ & $76-80(\mathrm{n}=10)$ \\
\cline { 2 - 6 } & $58.70 \pm .67$ & $62.90 \pm 1.37$ & $68.00 \pm 1.33$ & $73.40 \pm 1.35$ & $77.30 \pm 1.34$ \\
\hline Height $(\mathrm{cm})$ & $156.00 \pm 9.43$ & $155.40 \pm 5.97$ & $154.00 \pm 5.66$ & $149.80 \pm 6.86$ & $151.50 \pm 6.5$ \\
Weight $(\mathrm{kg})$ & $61.80 \pm 7.11$ & $60.40 \pm 7.55$ & $58.80 \pm 11.20$ & $56.70 \pm 5.42$ & $52.50 \pm 4.99$ \\
BMI $\left(\mathrm{kg} / \mathrm{cm}^{2}\right)$ & $25.61 \pm 4.03$ & $25.08 \pm 3.53$ & $24.75 \pm 4.00$ & $25.25 \pm 1.34$ & $22.85 \pm 1.19$ \\
\hline
\end{tabular}

BMI: Body Mass Index 
4 | J Korean Soc Phys Med Vol. 14, No. 4

Table 2. Evaluation of Low Extremity's Peak Torque

\begin{tabular}{|c|c|c|c|c|c|c|c|}
\hline Age (year) & & $\begin{array}{l}56-60 \\
(n=10)\end{array}$ & $\begin{array}{l}61-65 \\
(n=10)\end{array}$ & $\begin{array}{l}66-70 \\
(n=10)\end{array}$ & $\begin{array}{l}71-75 \\
(n=10)\end{array}$ & $\begin{array}{l}76-80 \\
(n=10)\end{array}$ & $p$ \\
\hline \multirow{4}{*}{$60^{\circ} / \mathrm{sec}$} & $\begin{array}{c}\text { Extension } \\
(\%)\end{array}$ & $\begin{array}{c}45.44 \pm 16.05 \\
(0 \%)\end{array}$ & $\begin{array}{c}40.58 \pm 4.21 \\
(-10.7 \%)\end{array}$ & $\begin{array}{c}35.64 \pm 2.48 \\
(-21.57 \%)\end{array}$ & $\begin{array}{c}27.81 \pm 2.34 \\
(-38.80 \%)\end{array}$ & $\begin{array}{c}24.84 \pm 6.65 \\
(-45.33 \%)\end{array}$ & $.000^{*}$ \\
\hline & $(\mathrm{Nm} / \mathrm{Kg})$ & $.76 \pm .32$ & $.69 \pm .15$ & $.62 \pm .11$ & $.49 \pm .04$ & $.47 \pm .13$ & \multirow{3}{*}{$.048^{*}$} \\
\hline & $\begin{array}{c}\text { Flexion } \\
(\%)\end{array}$ & $\begin{array}{c}23.42 \pm 9.24 \\
(0 \%)\end{array}$ & $\begin{array}{c}21.12 \pm 7.16 \\
(-9.82 \%)\end{array}$ & $\begin{array}{c}20.89 \pm 3.18 \\
(-10.80 \%)\end{array}$ & $\begin{array}{c}15.12 \pm 7.18 \\
(-35.44 \%)\end{array}$ & $\begin{array}{l}14.59 \pm 8.35 \\
(-37.70 \%)^{*}\end{array}$ & \\
\hline & $(\mathrm{Nm} / \mathrm{Kg})$ & $.39 \pm .18$ & $.36 \pm .13$ & $.37 \pm .09$ & $.26 \pm .11$ & $.28 \pm .17$ & \\
\hline \multirow{4}{*}{$180^{\circ} / \mathrm{sec}$} & $\begin{array}{c}\text { Extension } \\
(\%)\end{array}$ & $\begin{array}{c}33.43 \pm 8.37 \\
(0 \%)\end{array}$ & $\begin{array}{c}30.36 \pm 4.72 \\
(-9.18 \%)\end{array}$ & $\begin{array}{c}28.28 \pm 4.17 \\
(-15.41 \%)\end{array}$ & $\begin{array}{c}23.57 \pm 3.68 \\
(-29.49 \%)\end{array}$ & $\begin{array}{c}21.91 \pm 3.74 \\
(-34.46 \%)\end{array}$ & $.000^{*}$ \\
\hline & $(\mathrm{Nm} / \mathrm{Kg})$ & $.56 \pm .19$ & $.51 \pm .13$ & $.49 \pm .09$ & $.42 \pm .07$ & $.42 \pm .08$ & \multirow{3}{*}{$.028^{*}$} \\
\hline & $\begin{array}{c}\text { Flexion } \\
(\%)\end{array}$ & $\begin{array}{c}19.93 \pm 8.66 \\
(0 \%)\end{array}$ & $\begin{array}{c}19.62 \pm 4.32 \\
(-1.56 \%)\end{array}$ & $\begin{array}{c}19.71 \pm 2.99 \\
(-1.10 \%)\end{array}$ & $\begin{array}{c}14.80 \pm 4.90 \\
(-25.74 \%)\end{array}$ & $\begin{array}{c}14.06 \pm 6.09 \\
(-29.45 \%)\end{array}$ & \\
\hline & $(\mathrm{Nm} / \mathrm{Kg})$ & $.34 \pm .17$ & $.33 \pm .11$ & $.34 \pm .06$ & $.26 \pm .08$ & $.26 \pm .10$ & \\
\hline
\end{tabular}

*Significant Level : $p<.05$

Table 3. Post-Verification of Maximum Muscle Strength (Peak Torque) by Age Group

\begin{tabular}{ccccccc}
\hline & Age (year) & $56-60(\mathrm{n}=10)$ & $61-65(\mathrm{n}=10)$ & $66-70(\mathrm{n}=10)$ & $71-75(\mathrm{n}=10)$ & $76-80(\mathrm{n}=10)$ \\
\hline & $56-60$ & - & .353 & $.029^{*}$ & $.005^{*}$ & $.001^{*}$ \\
$60 \%$ sec & $61-65$ & .353 & - & $.004^{*}$ & $.000^{*}$ & $.000^{*}$ \\
Extension & $66-70$ & $.029^{*}$ & $.004^{*}$ & - & $.000^{*}$ & $.000^{*}$ \\
& $71-75$ & $.005^{*}$ & $.000^{*}$ & $.000^{*}$ & - & .393 \\
& $76-80$ & $.001^{*}$ & $.000^{*}$ & $.000^{*}$ & .393 &. \\
\hline & $56-60$ & - & .684 & .739 & $.029^{*}$ & .052 \\
$60^{\circ} / \mathrm{sec}$ & $61-65$ & .684 & - & .912 & .052 & .105 \\
Flexion & $66-70$ & .739 & .912 & - & $.043^{*}$ & .052 \\
& $71-75$ & $.029^{*}$ & $.052^{*}$ & $.043^{*}$ & - & .971 \\
\end{tabular}

"Significant Level: $p<.05$

2. Results of Muscle Strength and Endurance Evaluation

The peak torque and peak torque/body weight of the subjects at $60 \% \mathrm{sec}$ and $180 \% \mathrm{sec}$ are presented in Table 2 .

The results of the Post-hoc analysis by age group on the extension at $60 \% \mathrm{sec}$ showed that the 66-70, 71-75, and
76-80 age groups tended to have a significant reduction in muscle strength compared to the 56-60 and 61-65 age groups $(p<.05)$. In addition, the 71-75 and 76-80 age groups also showed significantly reduced muscle strength compared to the 66-70 age group $(p<.05)$. However, there was no significant difference between the 56-60 and 61-65 
Table 4. Post-Verification of Maximum Muscle Endurance (Peak Torque) by Age Group

\begin{tabular}{ccccccc}
\hline & Age (year) & $56-60(\mathrm{n}=10)$ & $61-65(\mathrm{n}=10)$ & $66-70(\mathrm{n}=10)$ & $71-75(\mathrm{n}=10)$ & $76-80(\mathrm{n}=10)$ \\
\hline \multirow{3}{*}{$180^{\circ} / \mathrm{sec}$} & $56-60$ & - & .393 & .143 & $.005^{*}$ & $.000^{*}$ \\
Extension & $61-65$ & .393 & - & .579 & $.002^{*}$ & $.000^{*}$ \\
& $66-70$ & .143 & .579 & - & $.009^{*}$ & $.003^{*}$ \\
& $71-75$ & $.005^{*}$ & $.002^{*}$ & $.009^{*}$ & - & .436 \\
& $76-80$ & $.000^{*}$ & $.000^{*}$ & $.003^{*}$ & .436 & - \\
\hline \multirow{3}{*}{ Flexion } & $56-60$ & - & .796 & .579 & .075 & .190 \\
& $61-65$ & .796 & - & .796 & $.043^{*}$ & $.019^{*}$ \\
& $66-70$ & .579 & .796 & - & $.023^{*}$ & $.009^{*}$ \\
& $71-75$ & .075 & $.043^{*}$ & $.023^{*}$ & - & .579 \\
& $76-80$ & .190 & $.019^{*}$ & $.009^{*}$ & .579 & - \\
\hline
\end{tabular}

"Significant Level: $p<.05$

age groups and the 71-75 and 76-80 age groups $(p>.05)$.

The post-verification results by age group for flexion at $60 \%$ sec showed that the $71-75$ age groups tended to show significant reduction in muscle strength compared to the 56-60, 61-65, and 66-70 age groups $(p<.05)$, but there was no significant difference in the other age groups $(p>.05)$ (Table 3).

The results of the post-hoc analysis by age group for extension at $180^{\circ} / \mathrm{sec}$ showed that the $71-75$ and $76-80$ age groups tended to show significant reduction in muscle strength compared to the 61-65 and 66-70 age groups $(p<.05)$, but there was no significant difference in the remaining age groups $(p>.05)$ (Table 4$)$.

The results of the post-hoc analysis by age group for flexion at $180^{\circ} / \mathrm{sec}$ showed that the $71-75$ and $76-80$ age groups tended to show significant reduction in muscle strength compared to the 61-65 and 66-70 age groups $(p<.05)$, but there was no significant difference in the remaining age groups $(p>.05)$ (Table 4$)$.

\section{Discussion}

In this study, the physical characteristics as well as muscle strength and endurance of the affected leg were analyzed using Biodex (Biodex System-4 pro, USA) based on classification into extension and flexion using female subjects who were 56-years-old or older and diagnosed with osteoarthritis (K-L II) on one knee.

In previous studies investigating isokinetic muscular function of the knee joint in Korean middle-aged women, which is similar to this study, Lee et al. (2001) measured maximum muscle strength at $60 \% \mathrm{sec}$ and $180 \% \mathrm{sec}$ and reported that the maximum strength of knee extension and flexion in those aged in their 40s was lower than that in 30-year-olds [25].

In addition, Kim and Kim [26], who measured muscle endurance and maximum muscle strength in Koreans over 50-years-old reported that the maximum muscle strength and endurance of the knee decreased with age and osteoarthritis. Their study supports the present study in that the maximum muscle strength of non-patients as well as the maximum muscle strength of patients diagnosed with osteoarthritis tended to decrease with age. Baert et al. [27] reported that the quadriceps femoris and femoral triceps muscle strength were weakened in patients with early or mid-term arthritis. The results of this study also showed strength weaknesses in age-related osteoarthritis of the same grade. Although reduction of knee strength in healthy 
elderly women according to age has been studied, it is hard to make a simple comparison due to differences in classification and measurement methods according to age. However, compared with another previous study [23], which examined the lower extremity muscle strength of elderly women between 60 and 80 years of age without any surgical experience, the mean peak torque of knee extension between 60 and 80 years was $54.35(\mathrm{Nm})$, which was higher than that of 55 to 60 -year-old subjects with the highest strength in this study. Elderly women's flexion peak torque was also $25.03(\mathrm{Nm})$, which is higher than the $23.42(\mathrm{Nm})$ value in this study. This suggests that even at the same age, muscle strength decreases due to degenerative arthritis.

According to Fleg et al. [28], the aging process in humans is divided into primary aging and secondary aging. In the primary aging process, physical activity through reduction of muscle function is reduced. In the second aging process, the overall deterioration of the body, such as flexibility and coordination, progresses in a complex manner. The results of this study show that the muscle strength of the legs is significantly reduced in patients over 70 years of age, compared with the 56-60 age group. In particular, muscle strength of the legs varied from $25.74 \%$ to $38.8 \%$ in the $71-75$ age group and from $29.45 \%$ to $45.33 \%$ in the over 76 age group. According to another study, the increase in the time of stance phase led by the progressed aging process causes reduction of balancing ability to increase the probability of falling injuries [29]. These problems are likely to have a significant impact on the reduction of activities in daily life. The results of this study can be used as basic data to recognize the importance of exercise programs to increase physical activity and muscle strength by evaluating the strength levels of lower body parts in subjects with osteoarthritis to prevent the aforementioned problems. In this study, however, there was no determination that muscle strength and exercise intensity prevented reduction of muscle strength caused by degenerative osteoarthritis. In addition, since the number of subjects per age group was small, further study will be needed with a larger number of subjects. However, the results of this study can be used as a basis for preventative exercise programs that can delay the secondary aging process related to neuro-responsiveness (flexibility and agility) by delaying the primary aging process (muscle strength reduction). This will have a positive effect on patients with osteoarthritis to maintain better movement range and quality of daily life.

\section{Conclusion}

As a result of this study, it was found that there was a significant correlation between muscle power and each group divided by age. The results can be used as basic data for increasing physical activity and muscle strength in women with knee osteoarthritis, applying a systematic exercise program considering the age-specific characteristics of elderly women with degenerative arthritis, as well as setting complementary training targets considering health level.

\section{Conflict of interest}

No conflicts of interest were declared.

\section{References}

[1] Kent-Braun JA, Ng AV, Young K. Skeletal muscle contractile and noncontractile components in young and older women and men. J Appl Physiol. 2000;88(2):662-68.

[2] Spirduso WW, Francis K, Eakin T, et al. Quantification of manual force control and tremor. J Mot Behav. 2005;37(3):197-210.

[3] Tani K, Kola I, Dhamaj F, et al. Physiotherapy Effects in Gait Speed in Patients with Knee Osteoarthritis. Open Access Maced J Med Sci. 2018;6(3):493-7. 
[4] Taruc-Uy RL, Lynch SA. Diagnosis and treatment of osteoarthritis. Prim Care. 2013;40(4):821-36.

[5] Ozdemir F, Tukenmez O, Kokino S, et al. How do marginal osteophytes, joint space narrowing and range of motion affect each other in patients with knee osteoarthritis. Rheumatol Int. 2006;26(6):516-22.

[6] Peter MM. Biomechanics of sport and Exercise (3rd ed). Champaign IL. Human Kinetics. 2013.

[7] Lennox IA, Cobb AG, Knowles J, et al. Knee function after patellectomy. A 12- to 48-year follow-up. J Bone Joint Surg Br. 1994;76(3):485-7.

[8] Kellgren JH, Lawrence JS. Radiological assessment of osteo-arthrosis. Ann Rheum Dis. 1957;16(4): 494-502.

[9] Coudeyre E, Jegu AG, Giustanini M, et al. Isokinetic muscle strengthening for knee osteoarthritis: A systematic review of randomized controlled trials with meta-analysis. Ann Phys Rehabil Med. 2016;59(3):207-15.

[10] Morrow JR, Mood D, Disch J, et al. Measurement and Evaluation in Human Performance (5th ed). Champaign IL. Human Kinetics. 2015.

[11] Sharma L, Cahue S, Song J, et al. Physical functioning over three years in knee osteoarthritis: role of psychosocial, local mechanical, and neuromuscular factors. Arthritis Rheum. 2003; 48(12):3359-70.

[12] Bascuas I, Tejero M, Monleon S, et al. Balance 1 year after TKA: correlation with clinical variables. Orthopedics. 2013;36(1):e6-12.

[13] Knoop J, Steultjens MP, van der Leeden M, et al. Proprioception in knee osteoarthritis: a narrative review. Osteoarthritis Cartilage. 2011;19(4):381-8.

[14] Blagojevic M, Jinks C, Jeffery A, et al. Risk factors for onset of osteoarthritis of the knee in older adults: a systematic review and meta-analysis. Osteoarthritis Cartilage. 2010;18(1):24-33.

[15] Lee S, Kwon Y, Lee N, et al. The Prevalence of Osteoarthritis and Risk Factors in the Korean Population: The Sixth Korea National Health and Nutrition Examination Survey (VI-1, 2013). Korean J Fam Med.
2019;40(3):171-5.

[16] Nagano Y, Ida H, Akai M,et al. Gender differences in knee kinematics and muscle activity during single limb drop landing. Knee. 2007;14(3):218-23.

[17] Sung PS, Lee DC. Gender differences in onset timing and activation of the muscles of the dominant knee during stair climbing. Knee. 2009;16(5):375-80.

[18] Petrella RJ, Lattanzio PJ, Nelson MG. Effect of age and activity on knee joint proprioception. Am J Phys Med Rehabil. 1997;76(3):235-41.

[19] Gillespie GN, Porteous AJ. Obesity and knee arthroplasty. Knee. 2007;14(2):81-6.

[20] Thompson KR, Mikesky AE, Bahamonde RE, et al. Effects of physical training on proprioception in older women. J Musculoskelet Neuronal Interact. 2003;3(3):223-31.

[21] Medina McKeon JM, Hertel J. Sex differences and representative values for 6 lower extremity alignment measures. J Athl Train. 2009;44(3):249-55.

[22] Hemmerich A, Brown H, Smith S, et al. Hip, knee, and ankle kinematics of high range of motion activities of daily living. J Orthop Res. 2006;24(4):770-81.

[23] Park IK, Bae YH. A Study on the Physical Strength of Middle-and-Old-Aged Female Gateball Players. Journal of The Korean Data Analysis Society. 2008;10(5):2953-66.

[24] Simoes LA, Dias JM, Marinho KC, et al. Relationship between functional capacity assessed by walking test and respiratory and lower limb muscle function in communitydwelling elders. Rev Bras Fisioter. 2010;14(1):24-30.

[25] Carregaro RL, Gentil P, Brown LE, et al. Effects of antagonist pre-load on knee extensor isokinetic muscle performance. J Sports Sci. 2011;29(3):271-78.

[26] Lee IM, Cho KH, Kim SY. A Comparative Analysis on Isokinetic Exercise Capacity to the adult Women's Knee Joint In Suwon Area. Bulletin of Dongnam Health University. 2001;19(1):73-81.

[27] Kim JH, Kim SB. Isokinetic Evaluation of the Knee Extensors and Flexors in the Normal Korean Adult. Journal 
of the Korean Academy of Rehabilitation Medicine. 1987;11(2):173-83.

[28] Baert IA, Jonkers I, Staes F, et al. Gait characteristics and lower limb muscle strength in women with early and established knee osteoarthritis. Clin Biomech (Bristol, Avon). 2013;28(1):40-7.
[29] Fleg JL, Morrell CH, Bos AG, et al. Accelerated longitudinal decline of aerobic capacity in healthy older adults. Circulation. 2005;112(5):674-82.

[30] Lee HJ, Lee CH, Yoo EY. Correlations Among the Berg Balance Scale, Gait Parameters, and Falling, in the Elderly. Phys Thera Korea. 2002;9(3):47-65. 